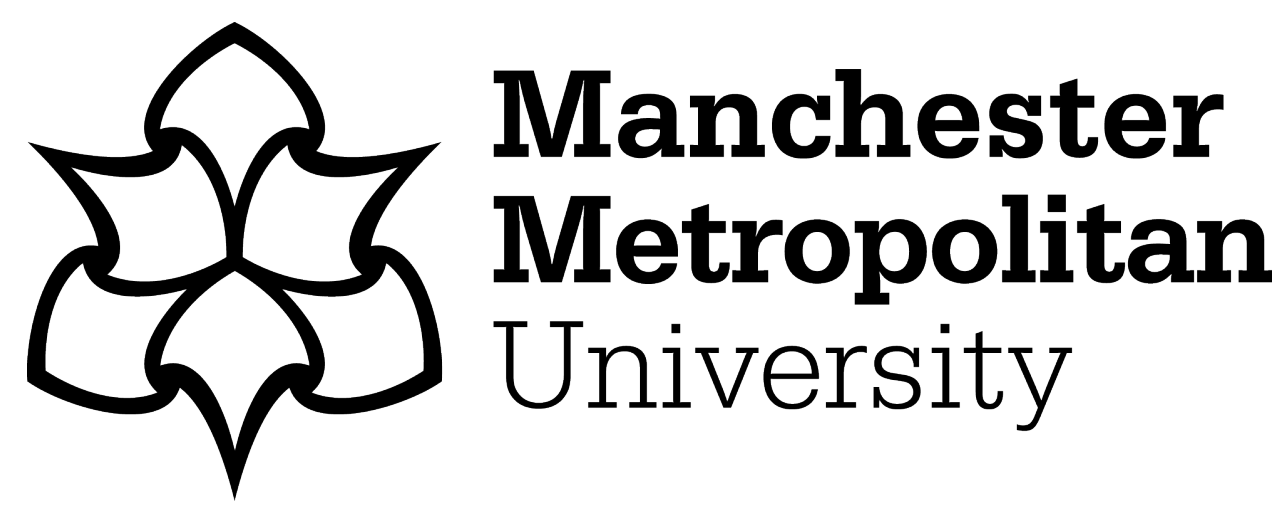

Decent, SP ORCID logoORCID: https://orcid.org/0000-0002-2272-6844, Pru, El, Simmons, MJH and Uddin, J (2018) On mathematical approaches to modelling slender liquid jets with a curved trajectory. Journal of Fluid Mechanics, 844. pp. 905-916. ISSN 0022-1120

Downloaded from: https://e-space.mmu.ac.uk/624562/

Version: Accepted Version

Publisher: Cambridge University Press

DOI: https://doi.org/10.1017/jfm.2018.221

Please cite the published version 


\title{
On mathematical approaches to modelling slender liquid jets with a curved trajectory
}

\author{
S. P. Decent ${ }^{1} \dagger$, E. I. Părău ${ }^{2}$, M. J. H. Simmons ${ }^{3}$ and J. Uddin ${ }^{4}$ \\ ${ }^{1}$ Department of Mathematics and Statistics, Lancaster University, Lancaster LA1 4YF, UK \\ ${ }^{2}$ School of Mathematics, University of East Anglia, Norwich NR4 7TJ, UK \\ ${ }^{3}$ School of Chemical Engineering, University of Birmingham, Birmingham B15 2TT, UK \\ ${ }^{4}$ School of Mathematics, University of Birmingham, Birmingham B15 2TT, UK
}

(Received xx; revised $\mathrm{xx}$; accepted $\mathrm{xx}$ )

Slender liquid jets that have a curved trajectory have been examined in a range of papers using a method introduced in Wallwork et al. (2000, 2002) and Decent et al. (2002), for jets that emerge from an orifice on the surface of a rotating cylindrical container, successfully comparing computational results to measurements arising from laboratory experiments. Wallwork et al. $(2000,2002)$ and Decent et al. (2002) based their analysis on the slenderness of the jet, and neglected the torsion of the centreline of the jet which is valid since in most situations examined the torsion is zero or small. Shikhmurzaev \& Sisoev (2017) used differential geometry and incorporated the torsion. This paper shows these two methods produce identical results at leading-order when the torsion is zero or when the torsion is $O(1)$, in an asymptotic framework based upon the slenderness of the jet, and shows that the method of Wallwork et al. (2000, 2002) and Decent et al. (2002) is accurate for parameters corresponding to scenarios previously examined and also when the torsion is $O(1)$. It is shown that the method of Shikhmurzaev \& Sisoev (2017) should be used when the torsion is asymptotically large or when the jet is not slender.

\section{Key words:}

\section{Mathematical approaches and approximations}

Curved liquid jets arise in a variety of industrial and manufacturing problems including propulsion systems for liquid jets in cross flow ( $\mathrm{Ng}$ et al. (2008)) and in agricultural sprays (Wong et al. (2004)). In recent years curved jets have found renewed interest in the production of nanofibres from centrifugal spinning (e.g. Noroozi et al. (2017), Zhmayev et al. (2015), Valipouri et al. (2015) and Riahi (2017)) which themselves have numerous applications in air filtration, optical sensors and tissue engineering to name just a few. A variety of careful studies of steady and time-dependent flows for curved liquid jets have been carried out by numerous groups, showing a wide variety of rich flow behaviours. Important studies include Entov \& Yarin (1984), Ribe (2004), Ribe et al. (2006), Panda et al. (2008), Marheineke \& Wegener (2009), Arne et al. (2010), Gramlich \& Piesche (2012) and Marheineke et al. (2016). There are other computational approaches which provide valuable descriptions of jet flows, particularly the lattice Boltzmann method, exemplified by Falcucci et al. (2010a,b, 2011a,b, 2013).

$\dagger$ Email address for correspondence: s.decent@lancaster.ac.uk 
One commonly studied application is when slender curved jets arise from a circular orifice on the outer surface of a rapidly rotating cylindrical container (where the axis of the rotating cylinder is vertical). The trajectory of the resulting liquid jet that emerges from the orifice follows the beginning of a spiral pathway that travels away from the rotating drum. Due to surface tension driven instability these jets rapidly break-up into droplets. This is often called prilling in industrial processes. This problem has been studied in a series of papers where the trajectory of the curved jets, their linear instability, nonlinear effects, and droplet break-up mechanisms were able to be examined computationally and the results compared successfully to experimental results across a range of physical and rheological parameters e.g. Wallwork (2002), Wallwork et al. (2000, 2002), Decent et al. (2002), Părău et al. (2006, 2007), Uddin et al. (2006, 2008a) and Gurney et al. (2010).

Wallwork et al. (2002) (henceforth labelled as W02) and Decent et al. (2002) (henceforth labelled as D02) introduced a multiple scales methodology to examine the unsteady dynamics of this slender curved jet as it breaks up into droplets. (W02 included rotation but neglected gravity in the model, while D02 included gravity and rotation.) One of these multiple scales is the arclength $s$, measured along the centreline of the curved jet, which is non-dimensionalised with respect to the radius of the rotating cylindrical container $s_{0}$. This lengthscale is much greater than the lengthscale $\bar{s}$ associated with the radius of the resulting droplet size or the wavelength of linear unstable waves that propagate along the jet and cause the jet to rupture. The method of multiple scales is used to examine linear instabilities so that $s=\varepsilon \bar{s}$, where $\varepsilon=a / s_{0}$ is a small parameter and $a$ is the radius of the orifice from which the jet emerges on the side of the rapidly rotating container. The resulting analysis assumes that $a \ll s_{0}$ and that the jet breaks up sufficiently far from the orifice such that the physical break-up length of the jet is much greater than the radius of the orifice $a$ i.e. the asymptotic analysis assumes that the jets are slender and the jet ruptures when $s=O(1)$. The experimental observations of W02, Wong et al. (2004), Partridge et al. (2005) and Hawkins et al. (2010) confirm that these simplifying assumptions are a reasonable starting point in order to describe the physical problem.

In order to deploy this multiple scales methodology to examine unstable linear waves travelling down the jet, it is first necessary to determine a steady solution for the curved jet which can be perturbed by the addition of these growing unstable linear waves. This involves the determination of steady trajectory equations that describe the curved slender jet in a steady state. It is these steady trajectory equations which are the main focus of this paper.

In W02 and D02, the coordinate used to describe these trajectory equations is the arclength $s$ measured along the centreline of the steady jet. In any cross-section of the jet, W02 and D02 also used plane polar coordinates in the radial and azimuthal directions $(n, \phi)$. W02 and D02 used base vectors $\mathbf{e}_{\mathbf{s}}, \mathbf{e}_{\mathbf{n}}, \mathbf{e}_{\phi}$ (defined in Wallwork (2002) and D02), where $\mathbf{e}_{\mathbf{n}}, \mathbf{e}_{\phi}$ lie in the cross-section of the jet. On the centreline $\mathbf{e}_{\mathbf{s}}$ is tangential to the centreline and $\mathbf{e}_{\mathbf{n}}, \mathbf{e}_{\phi}$ are perpendicular to the centreline, and this set of vectors are orthogonal unit vectors on the jet's centreline. The base vectors are shown in Figure 1. This coordinate system is inspired by the multiple scales inherent in the unsteady flow description of the jet, since on the short lengthscale $\bar{s}$ the coordinate system is locally cylindrically polar at leading-order in $\varepsilon$, and it gradually bends on the long lengthscale $s$.

It was pointed out in Entov \& Yarin (1984) that the base vectors at points away from the jet's axis are not orthogonal if the centreline of the curved jet has non-zero torsion. When gravity is neglected in the model, so that the centreline of the jet lies in a horizontal plane and thus the torsion of the jet's centreline is zero (as in W02, Partridge 


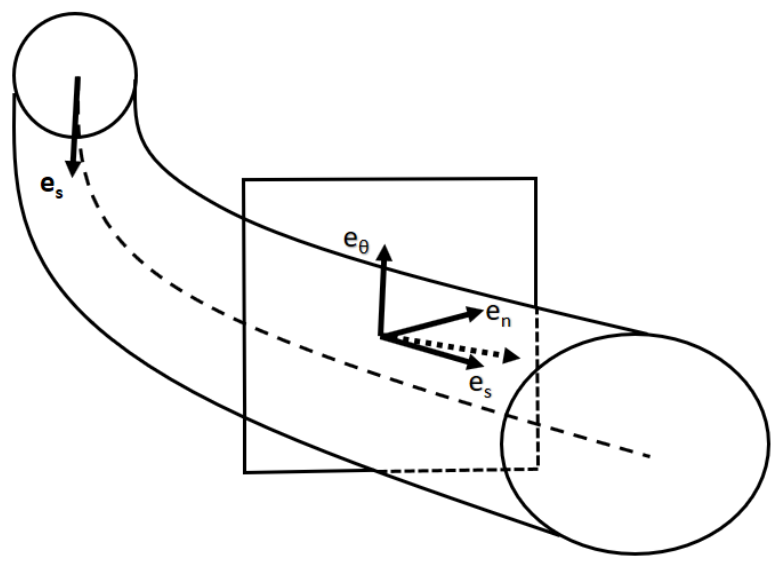

FiguRE 1. Diagram showing the base vectors in the jet. On the jet's centreline (dashed line), $\mathbf{e}_{\mathbf{s}}$ is always tangential to the centreline and $\mathbf{e}_{\mathbf{n}}, \mathbf{e}_{\phi}$ are perpendicular to the centreline, and this set of vectors are orthogonal unit vectors along all of the jet's centreline. The diagram shows an example of the vector $\mathbf{e}_{\mathbf{s}}$ that is tangential to the jet's centreline at a point at the far end of the portion of the jet shown in the diagram. However, these base vectors are not always orthogonal unit vectors at points away from the jet's axis, and such a situation is illustrated within the square box, which shows the base vectors at a point inside the jet and away from the jet's centreline. SS17 shows that the direction of $\mathbf{e}_{\mathbf{s}}$ can deviate away from the direction described in W02 and D02 at points away from the centreline, and this deviation is shown by the dotted vector which illustrates $\mathbf{e}_{\mathbf{s}}$ as determined in SS17. The deviation between the $\mathbf{e}_{\mathbf{s}}$ vector described by W02/D02 (vector shown by a solid-line arrow and labelled $\mathbf{e}_{\mathbf{s}}$ ) and the $\mathbf{e}_{\mathbf{s}}$ vector derived by SS17 (vector shown by a dotted-line arrow) is exaggerated in the Figure to aid visibility.

et al. (2005), Părău et al. (2006, 2007), Uddin et al. (2006, 2008a,b, 2009), Decent et al. (2009), Gurney et al. (2010), Hawkins et al. (2010), Uddin \& Decent (2012)) then the base vectors will remain exactly orthogonal throughout the jet. However, when gravity is included in the model, as in D02, then the jet's centreline falls out of the horizontal plane and has non-zero torsion. W02 and D02 treated the base vectors as though they were orthogonal unit vectors throughout the flow, which means that W02 and D02 provides an approximate methodology based on assuming that any terms neglected as a result will be insignificant given the asymptotic ordering because the jet is asymptotically slender. However, $\mathbf{e}_{\mathbf{s}}, \mathbf{e}_{\mathbf{n}}, \mathbf{e}_{\phi}$ are approximately orthogonal unit vectors when they are close to the jet's centreline even if the torsion is non-zero, and thus the approach adopted in W02 and D02 is an approximation that appears to be reasonable because the jet is asymptotically slender and the flow always remains asymptotically close to the centreline in W02, D02 and subsequent papers.

Shikhmurzaev \& Sisoev (2017) (henceforth labelled as SS17) has provided a mathematically formal framework for these curved jets, using differential geometry to tackle the problem rigorously, even when the centreline has non-zero torsion, and provides a rigorous framework for the treatment of the base vectors. This included showing that at points away from the centreline the direction of $\mathbf{e}_{\mathbf{s}}$ can deviate away from that described in W02 and D02, since W02/D02 forced $\mathbf{e}_{\mathbf{s}}$ to be parallel to the centreline at points away from the centreline while SS17 does not force a direction on $\mathbf{e}_{\mathbf{s}}$ but instead calculates it using differential geometry. Also SS17 showed that the length of the base vectors can vary away from the centreline. Consequently SS17 extends the applicability of the coordinate system into situations when the jet is not slender, and determines additional terms (not 
found in W02 and D02) which we show here are asymptotically small when the jet is slender.

This paper considers the above two approaches and compares the resulting steady models for a slender curved inviscid liquid jet, considering the cases when the curved jet's centreline has zero torsion and when it has non-zero torsion. In both cases this paper shows that the two approaches produce identical results at leading-order using slender jet asymptotic expansions (and so any terms neglected as a result of approximations are shown here to only appear at higher-order in the resulting asymptotic expansions). This paper demonstrates that the approximate methodology of W02 and D02 is useful and produces results to an acceptable level of approximation for slender curved liquid jets even when the jet's centreline has non-zero torsion. Given the ease of use of the approximate method, when compared to the rigorous approach of SS17, it provides a useful toolkit for tackling complex problems. This paper goes on to consider some asymptotic limits to understand when it is possible to use the approximate method and when it is necessary to use the rigorous method of SS17. This is important because the method of W02 (for centrelines with zero torsion) has been shown in a number of papers to be able to be implemented into situations where the liquid has complex rheology, has surfactants added, where temperature changes are incorporated, and where compound liquid jets are considered, in situations that incorporate linear instability and nonlinear mechanisms, and this has all been enabled because the method is straight-forward to use. If those complex applications are to be tackled in the future for situations where the centreline has non-zero torsion then it is necessary to understand whether D02 can be used as an accurate approximate methodology, and it is the primary result of this paper that this is in fact the case, thus opening out the future application of D02 into complex industrial problems for non-zero torsion as new applications arise.

\section{Slender jets}

In W02 and D02, a Cartesian coordinate system $x^{\prime}, y^{\prime}, z^{\prime}$ is used that rotates with the container where the $y^{\prime}$-axis is vertical and pointing upwards and the $x^{\prime}-z^{\prime}$ plane is horizontal, with the positive $x^{\prime}$-axis pointing away from the cylinder in a direction normal to the surface of the cylinder and the $z^{\prime}$-axis is tangential to the cylinder's surface. The origin of this coordinate system is located at the centre of the orifice from which the jet emerges on the surface of the rotating cylinder. The cylinder rotates about its central axis in an anti-clockwise direction when viewed from above. In non-dimensional terms, the radius of the rotating cylinder is 1 (i.e. lengths are non-dimensionalised with respect to $\left.s_{0}\right)$.

In SS17, a Cartesian coordinate system $x, y, z$ is used, that is considered here to rotate with the container, where the $z$-axis is vertical and pointing upwards and the $x-y$ plane is horizontal. The origin of this coordinate system is located on the axis of the rotating cylinder. For the two formulations to be equivalent, the centre of the orifice on the surface of the rotating cylinder is defined here to be at $x=1, y=z=0$ in the formulation of SS17, so that the $x$-axis also points through the centre of the orifice away from the rotating container's surface. The cylinder also rotates about its central axis in an anti-clockwise direction when viewed from above. Both Cartesian coordinate systems are right-handed.

In the formulations of W02 and D02, the centreline of the jet is described by nondimensional functions $X_{W a}(s), Y_{W a}(s), Z_{W a}(s)$ which describe the position of the jet's centreline with respect to $x^{\prime}, y^{\prime}, z^{\prime}$. The steady centreline of the jet is then described by the curve $x^{\prime}=X_{W a}(s), y^{\prime}=Y_{W a}(s)$ and $z^{\prime}=Z_{W a}(s)$. The leading-order steady jet 


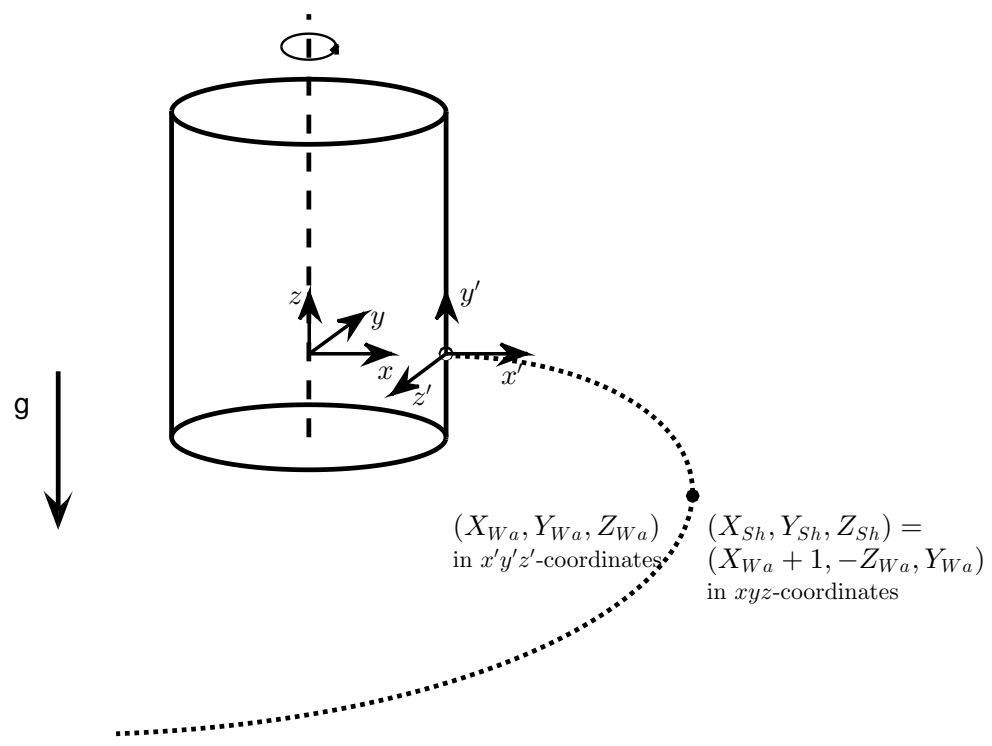

FiguRE 2. Comparison of the coordinate systems used in W02/D02 and SS17. The dotted curved line represents the jet's centreline for a liquid jet that emerges from the side of the rotating cylindrical container. The coordinate system of W02/D02 is $x^{\prime}, y^{\prime}, z^{\prime}$ and the coordinate system of SS17 is $x, y, z$. A point on the jet's centreline is shown that has coordinates $x^{\prime}=X_{W a}$, $y^{\prime}=Y_{W a}, z^{\prime}=Z_{W a}$ in W02/D02 and also $x=X_{S h}, y=Y_{S h}, z=Z_{S h}$ in SS17. The transformation between these coordinates is also shown for this point on the centreline. Both sets of Cartesian coordinates move with the rotating container, and the container rotates about its vertical axis in an anti-clockwise direction.

speed is denoted in W02 and D02 by $u_{0}(s)$ and the leading-order steady jet radius (which describes the location of the free-surface) is denoted by $R_{0}(s)$.

In the formulation of SS17, the centreline of the jet is described by $x=X_{S h}(s)$, $y=Y_{S h}(s)$ and $z=Z_{S h}(s)$ and the jet speed is denoted by $u_{\xi, 0}(s)$. (Since the Cartesian axes in W02/D02 and SS17 are aligned differently, we distinguish the position of the jet's centreline $(X, Y, Z)$ between the two formulations by writing subscripts "Wa" and "Sh" on those variables corresponding to W02/D02 and SS17 respectively. Also note that SS17 denotes the arclength using $\xi$ instead of $s$, so that $\xi=s$ in comparing W02/D02 and SS17, though we use $s$ as the arclength throughout this paper.) To transform between the two Cartesian systems note that $X_{S h}=X_{W a}+1$ so that the centre of the orifice is in the same place in both formulations (this is a translation of the origin in the Cartesian coordinate system between the two formulations). Also $Y_{S h}+Z_{W a}=0$ so that the direction of the Cartesian axes are aligned in the horizontal plane and $Z_{S h}=Y_{W a}$ so that the vertical axes are aligned. These coordinate systems are shown in Figure 2.

The Rossby, Froude and Weber numbers are $R b=U /\left(s_{0} \Omega\right), F r=U /\left(s_{0} g\right)^{1 / 2}$ and $W e=\rho U^{2} a / \sigma$ respectively, where $U$ is the exit speed of the jet at the orifice, $\Omega$ is the rate of rotation of the container, $\rho$ is the liquid's density, $\sigma$ is the surface tension of the liquid 
and $g$ is the acceleration due to gravity. The problem was examined in the inviscid case in W02 and D02 using a slender jet asymptotic expansion. The resulting non-dimensional trajectory equations derived in W02 and D02 are shown in the first column in Table 1 , where dashes on functions of $s$ denote differentiation with respect to $s$. In W02/D02 these equations are solved subject to the non-dimensionalised initial conditions (at the orifice) of $X_{W a}^{\prime}=R_{0}=u_{0}=1$ and $X_{W a}=Y_{W a}=Y_{W a}^{\prime}=Z_{W a}=Z_{W a}^{\prime}=0$ at $s=0$. The second column in Table 1 shows the leading-order trajectory equations from SS17.

W02 and D02 determined the equation in the first row of Table 1 using the nondimensionalised Euler's equation in the downstream direction combined with the normal stress boundary condition. The equation in the second row arises from the continuity equation and the kinematic boundary condition. The next two equations in Table 1 arise in W02 and D02 from Euler's equations in the radial and azimuthal directions in any cross-section of the jet combined with the normal stress boundary condition. The equation in the fifth row arises from the geometrical definition of the arclength $s$.

The following two subsections show that the equations in the two columns of Table 1 are identical both when gravity is included and when it is neglected.

\subsection{Trajectory equations including gravity}

D02 included gravity in the formulation, so that the centreline of the jet is a curve in three-dimensional space and the Froude number $F r=O(1)$. The trajectory equations in Table 1 arising from D02 and SS17 are identical, as is now shown, though this appears not to have been noticed in SS17.

There are two free non-dimensional constants in SS17 which need to be chosen here as $Q_{1}=1$ and $Q_{2}=1 / R b^{2}-2 / W e-1$. The jet speed functions are chosen to be identical in both formulations so that $u_{\xi, 0}=u_{0}$. Firstly it can be seen that the equations in columns 1 and 2 in Table 1 are identical for both of the top two rows using $R_{0}^{2} u_{0}=1$. (To show this, it is necessary to differentiate $R_{0}^{2} u_{0}=1$ with respect to $s$ and rearrange. This also gives the above mentioned formulae for $Q_{1}$ and $Q_{2}$ by comparing the equations in columns 1 and 2 , and this choice for $Q_{1}$ and $Q_{2}$ corresponds to ensuring that the initial conditions at the orifice are satisfied.) Next, by substituting the transformations between $\left(X_{W a}, Y_{W a}, Z_{W a}\right)$ and $\left(X_{S h}, Y_{S h}, Z_{S h}\right)$ into the equations in the third row of Table 1, it can be seen that the two equations in the third row are the same since $-Y_{W a}^{\prime \prime}\left(X_{W a}^{\prime 2}+Z_{W a}^{\prime 2}\right)+Y_{W a}^{\prime}\left(X_{W a}^{\prime} X_{W a}^{\prime \prime}+Z_{W a}^{\prime} Z_{W a}^{\prime \prime}\right)$ can be rearranged into $-Y_{W a}^{\prime \prime}$ because $X_{W a}^{\prime} X_{W a}^{\prime \prime}+Y_{W a}^{\prime} Y_{W a}^{\prime \prime}+Z_{W a}^{\prime} Z_{W a}^{\prime \prime}=0$ (from differentiating $X_{W a}^{\prime}{ }^{2}+$ $\left.Y_{W a}^{\prime}{ }^{2}+Z_{W a}^{\prime}{ }^{2}=1\right)$. Finally, it is straight-forward to see that the equations in the fourth and fifth rows of Table 1 are identical in each column. Therefore, the equations in the two columns in Table 1 are identical.

\subsection{Trajectory equations without gravity}

In the situation where rotation is sufficiently great that gravity can be neglected in the problem, then the jet does not fall before breaking up into droplets and this situation was examined in W02. The equations for W02 are shown in the first column of Table 1 for $Y_{W a}=0$ (so that the jet's centreline does not fall out of the plane $y^{\prime}=0$ ) and $F r=\infty$. (Note that the steady trajectory equations in W02 are written in a slightly different form to those shown in the first column of Table 1 for $Y_{W a}=0$ and $F r=\infty$, though it is straight-forward to rearrange the equations from the first column of Table 1 into the form shown in W02.) The equivalent trajectory equations from SS17 are shown in the second column of Table 1 for $Z_{S h}=0$ and $F r=\infty$.

Since this is just a special case of when gravity is included, the equations in the two 
TABLE 1. Comparison of the steady trajectory equations between W02/D02 and SS17. The equations in these two columns are shown in this paper to be identical using the transformations $u_{\xi, 0}=u_{0}, X_{S h}=X_{W a}+1, Y_{S h}+Z_{W a}=0$ and $Z_{S h}=Y_{W a}$.

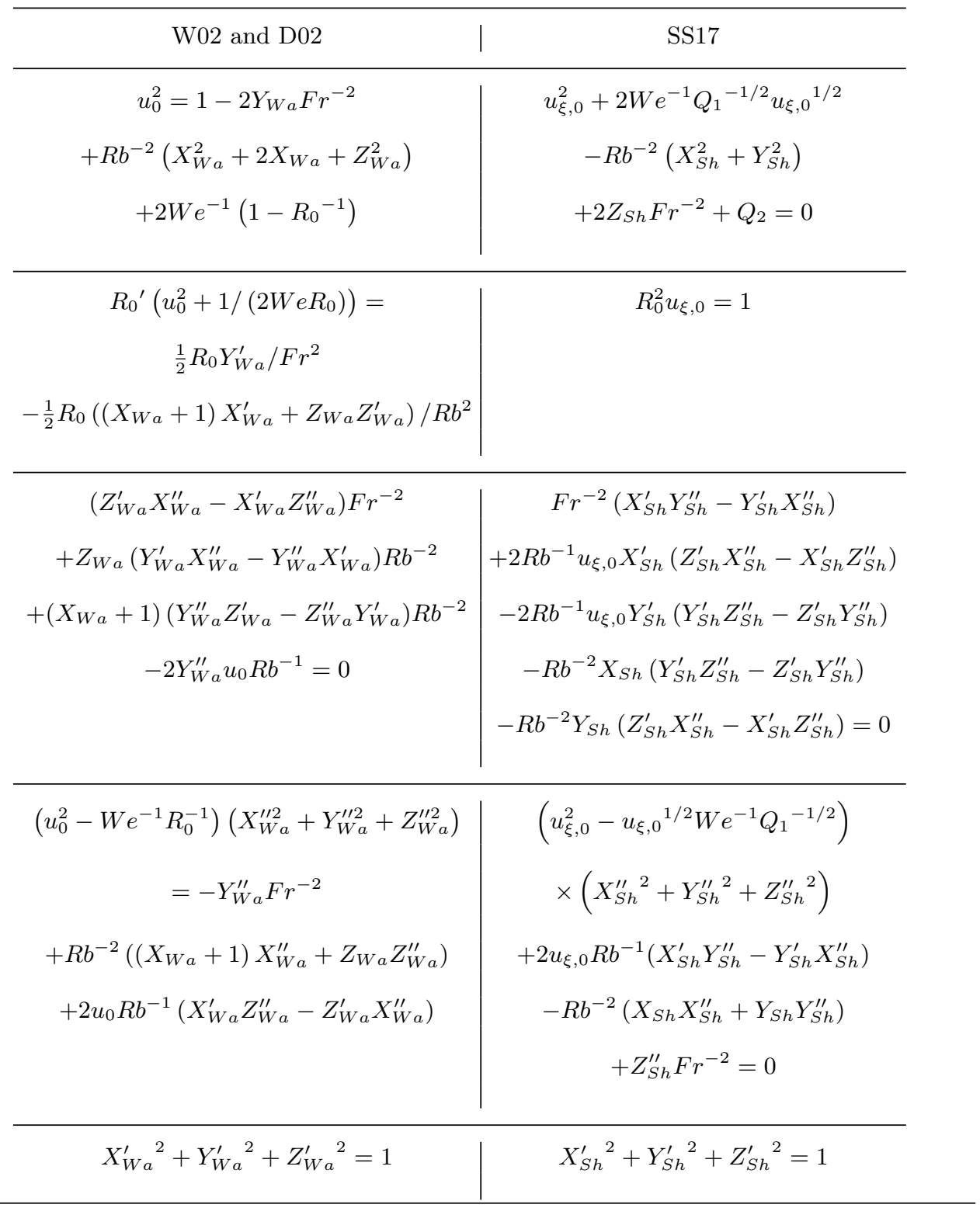

columns of Table 1 are again identical even when $Y_{W a}=Z_{S h}=0$ and $F r=\infty$ using the same arguments as in the previous subsection. The methodologies of W02/D02 and SS17 therefore produce identical results for the trajectory of a slender curved jet at leading-order when gravity is included (D02) and when it is not (W02). 


\subsection{Comparison of other equations and other models for slender jets}

In W02 and D02, the steady jet's radius from the centreline (i.e. the location of the undisturbed free-surface) is perturbed by a small linear travelling wave using the asymptotic expansion for the jet radius $R_{0}(s)+\delta \tilde{R}(\bar{s}, s, \bar{t})+\ldots$ where $\delta$ is a small parameter used to linearise the instability equations and $\bar{t}$ is a multiple-timescale defined in W02. Using this in SS17, W02 and D02, all three papers show that the curvature of the free-surface is equal to

$$
\frac{1}{R_{0}}-\delta\left(\frac{\partial^{2} \tilde{R}}{\partial \bar{s}^{2}}+\frac{\tilde{R}}{R_{0}^{2}}\right)+\ldots
$$

even when the torsion of the centreline is non-zero. Likewise, Euler's equations, the continuity equation, and the normal stress and the kinematic boundary conditions are the same at leading-order (at leading-order the jet is steady) and at $O(\delta)$ in W02 and SS17 (for $Y_{W a}=0$ and $F r=\infty$ ) when the torsion is zero, and are also identical at leading-order and at $O(\delta)$ in D02 and SS17 (for $F r=O(1)$ ) when the torsion is not equal to zero.

W02 and D02 go on to examine the linear instability of the jet in each case. The instability equations can be shown to be identical at leading-order to those produced by applying the same multiple-scales method of W02/D02 to SS17. That is because the instability arises on the short lengthscale $\bar{s}$.

The case examined in this paper is inviscid, but note that Wallwork (2002) and Decent et al. (2009) show that including viscosity does not change the trajectory equations from those determined in the inviscid case based upon a scaling where the Ohnesorge number $O h=\mu / \sqrt{\sigma a \rho}=O(1)$ and where $\mu$ is the dynamic viscosity of the liquid. The viscosity appears at leading-order in the equations for the unstable linear waves that propagate along the jet. The instability equations based upon W02 and D02 will again be the same as those based upon SS17 at leading-order when incorporating viscosity and the stress tensor. Subsequent papers such as Părău et al. (2006, 2007) and Uddin et al. (2006, $2008 a$ ) went on to examine the inclusion of nonlinear effects and additional physical effects such as surfactants and non-Newtonian liquid viscosity. Once again, in all of these situations at leading-order the two formulations will produce identical equations in both steady and unsteady cases. (Break-up and other complex unsteady phenomena can be significantly influenced by viscosity and the stress tensor e.g. Părău et al. (2007), Uddin et al. (2006) and Falcucci et al. (2013).)

\section{Discussion}

From Entov \& Yarin (1984), the coordinate system of W02/D02 is orthogonal everywhere when the torsion $\kappa_{2}$ of the centreline is equal to zero. From SS17, $\kappa_{2}=P / Q$ where

$$
\begin{gathered}
P=X_{S h}^{\prime}\left(Y_{S h}^{\prime \prime} Z_{S h}^{\prime \prime \prime}-Z_{S h}^{\prime \prime} Y_{S h}^{\prime \prime \prime}\right)+Y_{S h}^{\prime}\left(Z_{S h}^{\prime \prime} X_{S h}^{\prime \prime \prime}-X_{S h}^{\prime \prime} Z_{S h}^{\prime \prime \prime}\right) \\
+Z_{S h}^{\prime}\left(X_{S h}^{\prime \prime} Y_{S h}^{\prime \prime \prime}-Y_{S h}^{\prime \prime} X_{S h}^{\prime \prime \prime}\right)
\end{gathered}
$$

and

$$
Q=\left(Y_{S h}^{\prime} Z_{S h}^{\prime \prime}-Z_{S h}^{\prime} Y_{S h}^{\prime \prime}\right)^{2}+\left(Z_{S h}^{\prime} X_{S h}^{\prime \prime}-X_{S h}^{\prime} Z_{S h}^{\prime \prime}\right)^{2}+\left(X_{S h}^{\prime} Y_{S h}^{\prime \prime}-Y_{S h}^{\prime} X_{S h}^{\prime \prime}\right)^{2}
$$

In papers where gravity is neglected in the formulation, such as in W02, Părău et al. $(2006,2007)$ and Uddin et al. $(2006,2008 a)$, then $F r=\infty$ and $Z_{S h}=Y_{W a}=0$, so that 
$\kappa_{2}=0$ and the coordinate system used is orthogonal. When gravity is included, in D02, then $\kappa_{2} \neq 0$ and the coordinate system is not orthogonal.

Also note that jets observed in experiments and industrial prilling often rapidly breakup into droplets without falling significantly out of the horizontal plane, so that the vertical coordinate $Z_{S h}=Y_{W a} \approx 0$ at the break-up point in observations, so that $\kappa_{2} \neq 0$ but $\kappa_{2} \approx 0$ along many jets in prilling. However, as more applications of curved jets appear there will increasingly be demand to examine more complex situations.

Comparing D02 to SS17 it can be seen that when applying the slender jet asymptotic expansions there are terms that are different in the two papers which are $O(\varepsilon)$. The equations agree at $O(1)$ as has already been shown in this paper, but there are $O(\varepsilon)$ terms including ones proportional to $\kappa_{2}$ in (5.2)-(5.4) in SS17 which are absent in D02. In experiments such as those in W02, Wong et al. (2004), Partridge et al. (2005) and Hawkins et al. $(2010), \varepsilon \approx 0.01$. In the industrial applications such as prilling that have motivated the work (e.g. see Wallwork (2002), W02) $\varepsilon$ typically ranges from approximately 0.001 to 0.01 . Any corrections to measurable quantities caused by using SS17 instead of D02 will be of $O(\varepsilon)$ even if $\kappa_{2}=O(1)$. The main measurable outcome of the numerical simulations that subsequently use the solution of the trajectory equations are the predictions of the radius of droplets produced as a result of the surface tension driven instabilities. The droplet radius typically observed in experiments and in industrial applications is of the order of a few millimetres. Therefore the additional $O(\epsilon)$ terms derived by SS17 if implemented into a numerical simulation would produce corrections to the droplet radius of the order of at most $10^{-5} \mathrm{~m}$ which would be too small to be easily measured in an experiment (and other physical features neglected in the model such as air resistance will be more important that these small $O(\varepsilon)$ corrections). Furthermore, in the slender nanofibre applications (Noroozi et al. (2017)) these additional $O(\epsilon)$ terms from SS17 would cause corrections at only approximately the atomic scale.

It is also worth considering what happens for very long liquid threads in situations when the time-dependent instabilities are diminished so that the thread does not break-up due to surface tension, enabling $s$ to become very large. (The effects of surface tension would need to be very small. Such very long threads have not been observed in the experiments of W02 and subsequent papers but we can consider this scenario.) In the notation of $\mathrm{SS} 17, X_{S h}=r_{0} s^{N} \cos \left(\theta_{0} s^{M}\right)+\ldots, Y_{S h}=r_{0} s^{N} \sin \left(\theta_{0} s^{M}\right)+\ldots$ and $Z_{S h}=-\beta s+\ldots$ as $s \rightarrow \infty$, where $\beta>0, N, M, r_{0}$ and $\theta_{0}$ are constants. Examining the richest asymptotic balance available in the first equation listed in Table 1 gives that $N=1 / 2$. Examining the richest asymptotic balance available in the final equation listed in Table 1 gives that $M=1 / 2$. Therefore, the first equation listed in Table 1 gives that $u_{\xi, 0}=V \sqrt{s}+\ldots$ for $s \rightarrow \infty$, where $V^{2}=2 \beta / F r^{2}+r_{0}^{2} / R b^{2}$. The remaining equations in Table 1 give that $V^{2} \theta_{0}^{2}=4 / R b^{2}, \theta_{0}^{2}=2 \beta F r^{2} / R b^{2}$ and $r_{0}^{2} \theta_{0}^{2}+4 \beta^{2}=4$. Solving these equations gives that $\beta=-r_{0}^{2} F r^{2} /\left(4 R b^{2}\right)+\left(r_{0}^{4} F r^{4} /\left(16 R b^{4}\right)+1\right)^{1 / 2}$ where $r_{0}$ is a constant that is determined through the flow. The above equations then give that $\kappa_{2}=-2^{-1} \beta \theta_{0} s^{-1 / 2}+\ldots$ as $s \rightarrow \infty$, so that $\kappa_{2} \rightarrow 0$ as $s \rightarrow \infty$. So the coordinate system of D02 will tend towards becoming orthogonal as $s \rightarrow \infty$ for all parameter values.

The equations can also be solved for $s \rightarrow 0$. Using the notation of D02 and expanding in powers of $s$, the equations in Table 1 and the initial conditions give that

$$
X_{W a}=s-\frac{W e^{2}\left(R b^{2}+4 F r^{4}\right)}{6 F r^{4} R b^{2}(1-W e)^{2}} s^{3}+O\left(s^{4}\right),
$$




$$
\begin{gathered}
Y_{W a}=\frac{W e}{2 F r^{2}(1-W e)} s^{2}+\frac{W e^{3}}{R b^{2} F r^{2}(1-W e)^{2}(2 W e+1)} s^{3}+O\left(s^{4}\right), \\
Z_{W a}=\frac{W e}{R b(W e-1)} s^{2}-\frac{2 W e^{2}}{3 R b^{3}(1-W e)^{2}} s^{3}+O\left(s^{4}\right) \\
R_{0}=1-\frac{W e}{R b^{2}(2 W e+1)} s+O\left(s^{2}\right)
\end{gathered}
$$

and

$$
u_{0}=1+\frac{2 W e}{R b^{2}(2 W e+1)} s+O\left(s^{2}\right) .
$$

(The singularity at $W e=1$ is well known e.g. Keller \& Geer (1973), Baird \& Davidson (1962), Finnicum et al. (1993), Ramos (1996).) The transformations between D02 and $\mathrm{SS} 17$, and (3.1) and (3.2), give as $s \rightarrow 0$

$$
\kappa_{2}=-\frac{4 W e F r^{2}}{R b(2 W e+1)\left(R b^{2}+4 F r^{4}\right)}+O(s) .
$$

This is $O(1)$ for most parameters, though if $R b=O(\varepsilon)$ and $F r=O(1)$ (or e.g. if $R b=O(\varepsilon)$ and $F r=O(\varepsilon))$ then, for small $s, \kappa_{2}=O\left(\varepsilon^{-1}\right)$ so that the torsion is asymptotically large. (Varying $W e$ cannot make $\kappa_{2}$ asymptotically large if the other parameters are $O(1)$.) In this case the additional terms proportional to $\kappa_{2}$ identified in SS17 (which appear at $O(\varepsilon)$ in the asymptotic expansions) may become leading-order. Therefore, the scalings and approximations of D02 may break-down if $\kappa_{2}=O\left(\varepsilon^{-1}\right)$. Physically this corresponds to very high rotation rates in situations where gravity is also important. When $R b=O(\varepsilon)$ then the rotation rate of the cylinder $\Omega$ would need to be $O(U / a)$ which is very high. For example, in the laboratory experiments carried out in W02, rotation rates vary between 50 and 200 r.p.m., while $W e$ ranges from about 10 to $50, R b$ ranges from about 2 to 5 , and $F r$ ranges from about 2 to 3 , so that $\kappa_{2}$ is small at $s=0$ (from (3.8)). In contrast, if $R b$ was to be $O(\varepsilon)$ in the experiments of W02 then the rotation rate would need to have been around 10,000 r.p.m. which is well beyond the range of experiments carried out. (Note the variables in Table 1 vary rapidly close to the orifice at $s=0$ for $R b \ll 1$, as can be seen in (3.3) - (3.7), and there will be large gradients in the solutions for $R b \ll 1$.)

D02 will also break-down if the jet is not slender e.g. W02 and Wong et al. (2004) observed atomisation at the orifice in experiments for $W e \rightarrow 0$ when the jet ceases to be slender. (However, jets do not always shrink to zero for small $W e$ : see Falcucci et al. $(2010 b)$.)

\section{Conclusions}

In summary, the rigorous approach of SS17 has enabled the demonstration of the validity of the slender jet approach developed in W02 and D02 and has also enabled this detailed comparison between the two methodologies. It has been demonstrated that SS17 does not change the results of W02, D02 and subsequent papers, since the extra terms derived by SS17 are found to be asymptotically small in the case of slender curved jets and produce physical corrections too small to be easily measured. Note $\kappa_{2}$ is equal to zero in most of these previous papers (W02, Partridge et al. (2005), Părău et al. (2006, 2007), Uddin et al. (2006, 2008a,b, 2009), Decent et al. (2009), Gurney et al. 
(2010), Hawkins et al. (2010), Uddin \& Decent (2012)) and so the coordinate system used is orthogonal. Even when the torsion is non-zero and $O(1)$ (as in D02) the model of D02 remains accurate for a slender jet. The exception to this is when the torsion is asymptotically large close to the orifice when the method of D02 appears to break-down. Also if the jet is not slender then the formulation of W02/D02 is not likely to be accurate (since W02/D02 assumes that the jet is slender). Otherwise, the approximations made are accurate, and W02 and D02 describe the slender curved jet accurately in an easy to use approximate framework. This paper opens out the future application of D02 when $\kappa_{2}=O(1)$ into a wider variety of scenarios.

\section{REFERENCES}

Arne, W., Marheineke, N., Meister, A. \& Wegener, R. 2010 Numerical analysis of cosserat rod and string models for viscous jets in rotational spinning processes. Math. Mod. Meth. Appl. Sci. 20, 1941-1965.

Baird, M. H. I. \& Davidson, J. F. 1962 Annular jets - fluid dynamics. Chem. Eng. Sci. 17, $467-472$.

Decent, S. P., King, A. C., Simmons, M. J. H., Parau, E. I., Wallwork, I. M., Gurney, C. \& UdDin, J. 2009 The trajectory and stability of a spiralling liquid jet: Viscous theory. Appl. Math. Mod. 33, 4283-4302.

Decent, S. P., King, A. C. \& Wallwork, I. M. 2002 Free jets spun from a prilling tower. J. Eng. Math 42, 265-282.

Entov, V. M. \& YARIN, A. L. 1984 The dynamics of thin liquid jets in air. J. Fluid Mech. 140, 91-111.

Falcucci, G., Jannelli, E., Ubertini, S. \& Succi, S. 2013 Direct numerical evidence of stress-induced cavitation. J. Fluid Mech. 728, 362-375.

Falcucci, G., Succi, S. \& Ubertini, S. 2010 a Magnetically driven droplet break-up and vaporization: a lattice boltzmann study. J. Stat. Mech. 2010 (5), P05010.

Falcucci, G., Ubertini, S., Bella, G., De Maio, A. \& Palpacelli, S. $2010 b$ Lattice boltzmann modeling of diesel spray formation and break-up. SAE Int. J. Fuels Lubr. 3 (1), 582-593.

Falcucci, G., Ubertini, S., Biscarini, C. \& Di Francesco, S. 2011a Lattice boltzmann methods for multiphase flow simulations across scales. Comm. in Comp. Phys. 9, 269-296.

Falcucci, G., Ubertini, S., Chiappini, D. \& Succi, S. $2011 b$ Modern lattice boltzmann methods for multiphase microflows. IMA J. Appl. Math. 76, $712-725$.

Finnicum, D. S., Weinstein, S. J. \& Ruschak, K. J. 1993 The effect of applied pressure on the shape of a two-dimensional liquid curtain falling under the influence of gravity. $J$. Fluid Mech. 255, $647-665$.

Gramlich, S. \& Piesche, M. 2012 Numerical and experimental investigations on the breakup of particle laden liquid jets in the centrifugal field. Chemical Engineering Science 84, 408-416.

Gurney, C. J., Hawkins, V. L., Simmons, M. J. H. \& Decent, S. P. 2010 The impact of multi-frequency and forced disturbances upon drop distributions in prilling. Chem. Eng. Sci. 65, 3474-3484.

Hawkins, V. L., Gurney, C. J., Decent, S. P., Simmons, M. J. H. \& Uddin, J. 2010 Unstable waves on a curved non-newtonian liquid jet. Journal of Physics A: Mathematical and Theoretical 43, 055501.

Keller, J. B. \& Geer, J. 1973 Flows of thin streams with free boundaries. J. Fluid Mech. 59, $417-432$.

Marheineke, N., Liljegren-Sailer, B., Lorenz, M. \& Wegener, R. 2016 Asymptotics and numerics for the upper-convected maxwell model describing transient curved viscoelastic jets. Math. Models Meth. Appl. Sci. 26, $569-600$.

Marheineke, N. \& Wegener, R. 2009 Asymptotic model for the dynamics of curved viscous figres with surface tension. J. Fluid Mech. 622, $345-369$.

NG, C.-L., Sankarakrishnan, R. \& Sallam, K.A. 2008 Bag breakup of nonturbulent liquid jets in crossflow. Int. J. Multiphase Flow 34, 241-259. 
Noroozi, S., Alamdari, H., Arne, W., Larson, R. G. \& Taghavi, S. M. 2017 Regularized string model for nanofibre formation in centrifugal spinning methods. J. Fluid. Mech. 822, 202-234.

Panda, S., Marheineke, N. \& Wegener, R. 2008 Systematic derivation of an asymptotic model for the dynamics of curved viscous fibres. Math. Meth. Appl. Sci. 31, $1153-1173$.

PŭrăU, E. I., Decent, S. P., King, A. C., Simmons, M. J. H. \& Wong, D. 2006 Nonlinear travelling waves on a spiralling liquid jet. Wave Motion 43, 599-618.

PărăU, E. I., Decent, S. P., Simmons, M. J. H., Wong, D. \& King, A. C. 2007 Nonlinear viscous liquid jets from a rotating orifice. Journal of Engineering Mathematics 57, 159179.

Partridge, L., Wong, D. C. Y., Simmons, M. J. H., Părău, E. I. \& Decent, S. P. 2005 Experimental and theoretical description of the break-up of curved liquid jets in the prilling process. Chem. Eng. Res. Des. 83(A11), 1267-1275.

Ramos, J. J. 1996 Upward and downward annular liquid jets: conservation properties, singularities and numerical errors. Appl. Math. Mod. 20, $440-458$.

RiAhi, D. N. 2017 Modeling and computation of nonlinear rotating polymeric jets during forcespinning process. International Journal of Non-Linear Mechanics 92, 1-7.

Ribe, N. M. 2004 Coiling of viscous jets. Proc. R. Soc. Lond. A 460, 3223 - 3239.

Ribe, N. M., Habibi, M. \& Bonn, D. 2006 Stability of liquid rope coiling. Physics of Fluids 18, 084102 .

Shikhmurzaev, Y. D. \& Sisoev, G. M. 2017 Spiralling liquid jets: verifiable mathematical framework, trajectories and peristaltic waves. J. Fluid Mech 819, 352-400.

Uddin, J. \& Decent, S. P. 2012 Drop formation in rotating non-newtonian jets with surfactants. IMA J. Appl. Math. 77, 86-96.

Uddin, J., Decent, S. P. \& Simmons, M. J. H. 2006 The instability of shear thinning and shear thickening spiralling liquid jets: linear theory. ASME J. Fluids Eng. 128, 968-975.

Uddin, J., Decent, S. P. \& Simmons, M. J. H. 2008a The effect of surfactants on the instability of a rotating liquid jet. Fluid Dynamics Research 40, 827-851.

Uddin, J., Decent, S. P. \& Simmons, M. J. H. $2008 b$ Nonlinear waves along rotating nonnewtonian liquid jets. Int. J. Eng. Sci. 46, $1253-1265$.

Uddin, J., Decent, S. P. \& Simmons, M. J. H. 2009 Curved non-newtonian liquid jets. J. Fluids Eng. 131, 091203.

Valipouri, A., Ravandi, S. A. H., Pishevar, A. \& Parau, E. I. 2015 Experimental and numerical study on isolated and non-isolated jet behavior through centrifuge spinning system. Int. J. Multiphase Flow 69, $93-101$.

WALlwork, I. M. 2002 The trajectory and stability of a spiralling liquid jet. PhD thesis, School of Mathematics and Statistics, University of Birmingham.

Wallwork, I. M., Decent, S. P. \& King, A. C. 2000 The trajectory and stability of a spiralling liquid jet. In Proc. IUTAM Symp. Free-Surface Flows (ed. A.C. King \& Y.D. Shikhmurzaev). Kluwer.

Wallwork, I. M., Decent, S. P., King, A. C. \& Schulkes, R. M. S. M. 2002 The trajectory and stability of a spiralling liquid jet. part 1. inviscid theory. J. Fluid Mech 459, 43-65.

Wong, D., Simmons, M. J. H., Decent, S. P., PărăU, E. I. \& King, A. C. 2004 Breakup dynamics and drop size distributions created from spiralling liquid jets. International Journal of Multi-Phase flow 30, 499-520.

Zhmayev, Y., Divvela, M. J., Ruo, A., Huang, T. \& Joo, Y. L. 2015 The jetting behavior of viscoelastic boger fluids during centrifugal spinning. Physics of Fluids 27, 123101. 